\title{
Impact of early versus delayed androgen- deprivation therapy on survival outcomes of patients with localized prostate cancer who underwent radical prostatectomy and later developed metastasis: Can we define a PSA threshold?
}

Hyun Kyu Ahn

Yonsei University College of Medicine

Kwang Suk Lee

Yonsei University College of Medicine

Daeho Kim

Yonsei University College of Medicine

Koon Ho Rha

Yonsei University College of Medicine

Sung Joon Hong

Yonsei University College of Medicine

\section{Byung Ha Chung}

Yonsei University College of Medicine

Kyo Chul Koo ( $\nabla$ gckoo@yuhs.ac)

Yonsei University College of Medicine https://orcid.org/0000-0001-7303-6256

Research article

Keywords: androgens, neoplasm metastasis, prostatic neoplasm, survival

Posted Date: March 24th, 2020

DOI: https://doi.org/10.21203/rs.3.rs-18688/v1

License: @ (i) This work is licensed under a Creative Commons Attribution 4.0 International License. Read Full License 


\section{Abstract}

Background: The benefits of early administration of androgen-deprivation therapy (ADT) in patients with prostate-specific antigen (PSA)-only recurrent prostate cancer (PCa) following radical prostatectomy (RP) are controversial. We investigated the impact of early versus delayed ADT on survival outcomes in patients with non-metastatic, localized or locally advanced PCa who received radiation therapy following RP and later developed distant metastasis.

Methods: A retrospective analysis was performed on 69 patients with non-metastatic, localized or locally advanced PCa who received radiation therapy following RP and later developed distant metastasis between January 2006 and December 2012. Patients were stratified according to the level of PSA at which ADT was administered ( $<2 \mathrm{ng} / \mathrm{mL}$ vs. $\geq 2 \mathrm{ng} / \mathrm{mL}$ ). Study endpoints were progression to castrationresistant prostate cancer (CRPC)-free survival and cancer-specific survival (CSS) assessed by KaplanMeier analysis and Cox-regression models.

Results: Patients were stratified according to the level of PSA at which ADT was administered $(<2 \mathrm{ng} / \mathrm{mL}$ vs. $\geq 2 \mathrm{ng} / \mathrm{mL}$ ) based on the Youden sensitivity analysis. Delayed ADT at PSA $\geq 2 \mathrm{ng} / \mathrm{mL}$ was an independent prognosticator of cancer-specific mortality $(p=0.047)$, and a marginally significant prognosticator of progression to CRPC ( $p=0.051)$. During the median follow-up of 81.0 (IQR 54.2-115.7) months, patients who received early ADT at PSA $<2 \mathrm{ng} / \mathrm{mL}$ had significantly higher CSS rates than patients who received delayed ADT at PSA $\geq 2 \mathrm{ng} / \mathrm{mL}(p=0.002)$. Progression to CRPC-free survival was comparable between the two groups $(p=0.331)$.

Conclusions: Early ADT at the PSA level of less than $2 \mathrm{ng} / \mathrm{mL}$ confers CSS benefits in patients with localized or locally advanced PCa previously treated with RP.

\section{Background}

Radical prostatectomy (RP) is used as a curative therapy for patients with prostate cancer (PCa). RP has been shown to confer excellent oncological control and long-term survival for localized PCa by reducing the risk of local tumor progression and metastasis [1]. RP can be used as a therapeutic option for various stages of $\mathrm{PCa}$, from localized to high-risk disease [2]. Furthermore, recent evidence suggests a survival benefit when RP is included as part of multimodal therapy in patients with oligometastatic PCa [3].

Androgen deprivation therapy (ADT) is the standard therapy for patients with metastatic PCa and can also be employed as part of multimodal therapy following RP. For patients exhibiting adverse pathological features, ADT can be used as an adjuvant therapy with radiation therapy (RT) to improve cancer-specific survival (CSS) and overall survival (OS) [4-8]. ADT can also be administered after prostate-specific antigen (PSA) recurrence with RT, depending on the presence of metastasis, as a salvage treatment [9]. However, the optimal value of PSA and the disease landscape in which ADT should be administered are still a matter of debate. 
Early initiation of ADT may be superior to delayed ADT in terms of short-term oncological outcomes; however, the benefit of early ADT is unclear regarding CSS or OS outcomes [2]. Adverse effects of ADT, including hot flashes, sexual dysfunction, and osteoporosis, cannot be overlooked. Moreover, the advent of non-metastatic castration-resistant disease may be attributed to early administration of ADT [10]. On the other hand, $12 \%$ of patients receiving RP for localized or locally advanced PCa are destined to develop metastasis during the median follow-up of 2.2 years, and early ADT may be a feasible treatment option to delay the onset of metastatic progression $[11,12]$.

Contemporary guidelines do not indicate the optimal timing for administering ADT following RP and state that treatment should be individualized depending on PSA kinetic parameters such as PSA doubling time (PSADT), PSA velocity (PSAV), patient anxiety, underlying comorbidities, and life expectancy [2]. Indeed, this disease spectrum poses uncertainty for both patients and physicians and warrants investigation. The primary endpoint of our study was the impact of early versus delayed ADT on survival outcomes in patients with localized or locally advanced PCa who received RP and later developed metastasis. The secondary study endpoint was the definition of a specific level of PSA-related parameters that can be utilized to select candidates for early initiation of ADT

\section{Methods}

\subsection{Patient Selection}

This multicenter study evaluated data from 923 consecutive patients with non-metastatic, localized or locally advanced PCa who received adjuvant or salvage radiation therapy for PSA-only recurrence following RP between January 2006 and December 2012. Among these patients, were evaluated for 69 (7.5\%) patients who later developed distant metastasis were selected for analysis (Fig. 1). The study's protocol was approved by the institutional ethics committee (2017-0186-001).

\subsection{Data Collection}

The patients' clinicopathological characteristics were retrieved from the institutional electronic medical records database. The variables included age, body mass index, Eastern Cooperative Oncology Group Performance Status Scale, National Comprehensive Cancer Network (NCCN) risk category, time to PSA recurrence, pathological Gleason score and stage, preoperative PSA level, PSA nadir at RP, PSA level at ADT initiation, PSAV and PSADT before ADT, PSA nadir after ADT, and time to PSA nadir.

Prostate cancer staging was determined according to the $7^{\text {th }}$ version of the American Joint Committee on Cancer TNM system. Castration-resistant prostate cancer (CRPC) was defined as the progression of disease or an increase in serum PSA using the Prostate Cancer Working Group 2 criteria [13]. The progression of disease was diagnosed based on a continuous increase in serum PSA levels, new symptom development, or a metastatic lesion detected during ADT using bone scanning, computed tomography, magnetic resonance imaging, or positron emission tomography. 
For all patients, the status of survival and cause of death were investigated using institutional electronic medical records, the National Cancer Registry Database, or the Social Security Death Index. Death was attributed to PCa if evidence of progressive metastatic CRPC was present, PCa was listed on the death certificate as the cause of death, or if the patient died of complications of PCa treatment.

\subsection{Treatments}

Robot-assisted laparoscopic RP was recommended for patients who were determined to be reasonable surgical candidates and desired surgical treatment. Surgery was performed with the extent of pelvic lymph node dissection being based upon the risk category of the patient.

RT was delivered to the prostatic fossa with defined margins according to the guidelines of the European Organization for Research and Treatment of Cancer [14]. At both institutions, RT consisted of 3D conformal radiation therapy from 2000 to 2007 and intensity-modulated external beam RT from 2007 to 2016. The median RT dose delivered was 6300 cGy (IQR 6300 - 6300 cGy).

ADT included luteinizing hormone-releasing hormone (LHRH) agonists only or combined androgen blockade. LHRH agonist was administered by injection every three or six months according to the physician's discretion.

All patients received the standard-of-care according to contemporary guidelines until death or the last follow-up.

\subsection{Study Endpoints}

The primary endpoint was progression to CRPC-free survival and CSS. The secondary endpoint was specific levels of PSA-related parameters that can be utilized to select candidates for the initiation of ADT.

\subsection{Statistical analysis}

The chi-square test and ANOVA were used to compare two or more variables, and the Mann-Whitney U test was used for the analysis of continuous variables. Survival analysis was evaluated and compared using the Kaplan-Meier method and compared with the log-rank test. The prognostic significances of PSAV and PSADT before ADT, PSA at ADT, and PSA nadir after ADT were dichotomized at $2 \mathrm{ng} / \mathrm{mL} /$ year, 12 months, $2 \mathrm{ng} / \mathrm{mL}$, and $1 \mathrm{ng} / \mathrm{mL}$, respectively. These optimal cut-off values were based on predefined values and according to sensitivity analysis using Youden's Index. Multivariate analyses were performed with Cox-proportional hazards regression models to adjust for potential confounders. All statistical analyses were performed using IBM SPSS software (version 21.0; IBM Corporation, Armonk, NY, USA). Differences with a $p$-value of $<0.05$ were considered statistically significant.

\section{Results}

\subsection{Patient characteristics}


Patient demographics and clinicopathological features of groups stratified by the PSA level at ADT of 2 $\mathrm{ng} / \mathrm{mL}$ are presented in Table 1. There were no significant differences between the two groups regarding patient age, body mass index, performance status, preoperative PSA level, NCCN risk category, pathological Gleason score and stage, type of RT, and PSA velocity and PSA doubling time before ADT.

\subsection{Survival Prognosticators}

On multivariable Cox regression analyses, delayed ADT (PSA $\geq 2 \mathrm{ng} / \mathrm{mL})$ was an independent prognosticator of cancer-specific mortality $(p=0.047$; Table 2$)$, and a marginally significant prognosticator of progression to CRPC ( $p=0.051$; Table 3$)$.

Patient age, body mass index, grade and stage, preoperative PSA, PSA nadir at RP, PSAV and PSADT prior to $A D T$, and time to PSA nadir following ADT were not independently associated with progression to CRPC and cancer-specific mortality.

\subsection{Survival outcome}

Survival outcomes were compared according to the level of PSA at ADT, stratified at $2 \mathrm{ng} / \mathrm{mL}$ (Table 4). During the median follow-up of 81.0 (IQR 54.2-115.7) months, patients who received ADT at PSA $<2$ $\mathrm{ng} / \mathrm{mL}$ had significantly higher CSS rates than men who received ADT at PSA $\geq 2 \mathrm{ng} / \mathrm{mL}(p=0.002$; Fig. 2). Progression to CRPC-free survival was comparable between the two groups ( $p=0.331$; Fig. 3 ).

\section{Discussion}

ADT is a treatment option for all stages of PCa, from localized disease with a high risk of recurrence to castration-resistant disease [15]. Following RP, ADT can be used as an adjuvant therapy combined with RT to maximize survival outcomes in patients exhibiting adverse pathological features, including pT3 disease, positive surgical margin, Gleason score 8-10, and seminal vesicle invasion. The results of previous studies have suggested that the use of ADT in this clinical scenario may improve CSS and OS [4-9]. ADT can also be administered with RT at biochemical recurrence, depending on the presence of metastasis, as a salvage treatment option, which may result in clinical benefit [9]. However, the optimal level of PSA at which ADT should be administered is still controversial. In this study, we observed that delaying ADT following PSA elevation beyond $2 \mathrm{ng} / \mathrm{mL}$ was associated with increased risk of progression to CRPC and cancer-specific mortality.

In our study, a PSA cut-off value of $2 \mathrm{ng} / \mathrm{mL}$ based on Youden sensitivity analysis was revealed to be a prognosticator for CSS in patients with PSA-only recurrent PCa following RP. Previous studies report varying indications for initiating ADT following RP. Amling et al. suggested that a PSA value of greater than $0.4 \mathrm{ng} / \mathrm{mL}$ should be used to define PSA recurrence, since this cut-off point is associated with a significantly increased risk of biochemical and/or clinical progression over the following three years [16]. Freedland et al. reported that the risk of the need for secondary treatment following PSA recurrence depends on the cut-off value of PSA. On the basis of the finding that patients with a postoperative PSA 
greater than $0.2 \mathrm{ng} / \mathrm{mL}$ had a 100\% 3-year risk of PSA progression, this cut-off point was suggested to be an appropriate indicator to initiate treatment [17]. Siddiqui et al. suggested that adjuvant ADT within 90 days following after RP improves CSS and systemic progression-free survival in node-negative patients. However, there were no significant differences in systemic progression-free survival or CSS between patients started on ADT at PSA values of $0.4,1.0$, and $2.0 \mathrm{ng} / \mathrm{mL}$ [18]. A randomized trial investigated the efficacy of immediate ADT versus delayed ADT of at least two years after randomization among patients with PSA recurrence following curative therapy and who were considered ineligible for curative treatment [19]. In the overall group, immediate ADT was associated with improved OS and time to clinical progression. However, there was no significant improvement of OS in the subgroup analysis, which included patients with PSA recurrence following curative therapy. The common limitation of these studies was that not all patients had received RT as multimodal therapy, as suggested by contemporary guidelines. In our study, all patients were treated with RT in an adjuvant or salvage setting according to the guidelines, which implies the generalizability of our data.

In our study, PSAV, PSADT, and Gleason score were not independently associated with survival endpoints. On the other hand, several studies have recommended that these parameters be utilized to decide on the timing of ADT at PSA-recurrence following RP [19-29]. Van den Bergh, et al. reported in a systematic review that early ADT cannot be recommended as the standard-of-care in the setting of PSA recurrence or local recurrence, and that ADT should be reserved for patients with the highest risk of disease progression, defined as short PSADT of less than 6-12 months or Gleason score of greater than 8 [21]. Algarra, et al. reported seminal vesical involvement and PSAV of greater than $0.84 \mathrm{ng} / \mathrm{mL} /$ year, in addition to PSADT, to be adverse features associated with disease progression in patients who received ADT at PSA recurrence [22]. In a cohort of patients with mainly high-risk disease, faster PSADT, higher Gleason score, and early intervention were associated with a lower risk of CSS [25]. The significance of PSADT was consistent in patients receiving intermittent ADT, in which a PSADT and PSA nadir of less than $1 \mathrm{ng} / \mathrm{mL}$ during the first cycle was associated with improvement in CRPC-free survival [24]. On the other hand, studies have reported higher PSA and PSA nadir after starting ADT, rather than PSADT or PSAV, to be significant indicators for CSS $[28,29]$. As seen in these studies mentioned above, the parameters utilized for clinical endpoints were inconsistent, which defers a definite conclusion. Moreover, various cut-off points were used for stratification of PSADT or PSAV, if they were not evaluated as continuous variables. To the best of our knowledge, the present study is the first to suggest an optimal cut-off point of PSA to initiate ADT in order to confer CSS benefit in patients who underwent RT after PSA-only recurrence. Our results are meaningful because the patients included in our study were a homogeneous group who were treated with RT and standard care according to contemporary guidelines until death or last follow-up.

Based on accumulating evidence, there has been a paradigm shift in considering aggressive treatments targeted at both the primary tumor and metastatic lesions of PCa to avoid or delay the need for palliative treatments and to achieve maximal survival benefit [3]. PSA recurrence is the most common pattern of disease relapse following RP, observed in up to $35 \%$ of patients with clinically localized PCa [30, 31]. In an era of aggressive treatments such as RP for the treatment of oligometastatic $\mathrm{PCa}$, it is certain that more 
patients will experience post-operative PSA recurrence and would be candidates for ADT. Whether ADT alone or in combination with systemic chemotherapy is considered in this clinical scenario, we believe that our findings provide relevant evidence for decision-making and patient stratification in future clinical trials.

The strengths of our study are the inclusion of detailed PSA kinetic data, comorbidities, performance status, and clinicopathological data that were available for all patients. At the same time, we acknowledge several limitations: first, our study is limited by its retrospective design. Sampling intervals used to estimate PSA kinetics were not standardized. Second, the potential existence of bias regarding subgroup differences may have confounded the results, although there were no statistically significant differences between the two groups stratified by the level of PSA at ADT. Third, patient and physician preferences affected the implementation of specific treatments. Finally, differences in adverse effects and quality-of-life following ADT were not investigated. However, a notable finding of our study was that the early administration of ADT was not associated with an early advent of non-metastatic CRPC.

\section{Conclusions}

Early ADT at the PSA level of less than $2 \mathrm{ng} / \mathrm{mL}$ confers a CSS benefit in patients with localized or locally advanced PCa previously treated with RP. Future larger-scale analyses are warranted to validate our results.

\section{Abbreviations}

ADT: androgen-deprivation therapy

CRPC: castration-resistant prostate cancer

CSS: cancer-specific survival

NCCN: National Comprehensive Cancer Network

OS: overall survival

PCa: prostate cancer

PSA: prostate-specific antigen

PSADT: PSA doubling time

PSAV: PSA velocity

RP: radical prostatectomy

RT: radiation therapy 


\section{Declarations}

\section{Ethics approval and consent to participate}

This study was approved by the Yonsei University Health System Institutional Review Board (2017-0186001). Informed consent was waived from the Yonsei University Health System Institutional Review Board since patients' information was collected during the routine clinical practice and patients were identified by anonymized investigator-generated code not linkable to their personal data. The same Institutional Review Board granted access to the institutional databases used in this study.

Consent for publication: Not required

Availability of data and materials: The dataset analyzed during the current study is available from the corresponding author on reasonable request.

Competing interests: The authors declare that they have no competing interests.

Funding : This study was supported by the Young Researcher Program Grant from the National Research Foundation of Korea (NRF-2017R1C1B5017516). The funding body had no active role in any stage of the study, including: design, data collection, analysis, interpretation of data, and writing the manuscript.

\section{Author's contributions}

Study concept and initial design: HKA, KCK; Acquisition of data: HKA, KSL, DK; Data analysis: HKA, KSL; Manuscript writing: HKA, KCK; Critical revision for important intellectual content: BHC, KHR, SJH. All authors have participated sufficiently in the work to take public responsibility for appropriate portions of the content, and have given final approval of the version to be published. All authors agreed to be accountable for all aspects of the work in ensuring that questions related to the accuracy or integrity of any part of the work are appropriately investigated and resolved.

Acknowledgments : None.

\section{References}

[1] Tan L, Wang LL, Ranasinghe W, Persad R, Bolton D, Lawrentschuk N, et al. Survival outcomes of younger men (< 55 years) undergoing radical prostatectomy. Prostate Int. 2018;6:31-5.

[2] Mohler JL, Antonarakis ES, Armstrong A J, D'Amico A V, Davis BJ, Dorff T, et al. Prostate Cancer, Version 2.2019, NCCN Clinical Practice Guidelines in Oncology. J Natl Compr Canc Netw. 2019;17:479505.

[3] Koo KC, Dasgupta P. Treatment of Oligometastatic Hormone-Sensitive Prostate Cancer: A Comprehensive Review. Yonsei Med J. 2018; 59:567-79. 
[4] Bolla M, Collette L, Blank L, Warde P, Dubois JB, Mirimanoff RO, et al. Long-term results with immediate androgen suppression and external irradiation in patients with locally advanced prostate cancer (an EORTC study): a phase III randomised trial. Lancet. 2002;360:103-6.

[5] Ahn S, Lee M, Jeong CW. Comparative quality-adjusted survival analysis between radiation therapy alone and radiation with androgen deprivation therapy in patients with locally advanced prostate cancer: a secondary analysis of Radiation Therapy Oncology Group 85-31 with novel decision analysis methods. Prostate Int. 2018;6:140-4.

[6] Pilepich MV, Winter K, Lawton CA, Krisch R E, Wolkov HB, Movsas B, et al. Androgen suppression adjuvant to definitive radiotherapy in prostate carcinoma-long-term results of phase III RTOG 85-31. Int $\mathrm{J}$ Radiat Oncol Biol Phys. 2005;61:1285-90.

[7] Warde P, Mason M, Ding K, Kirkbride P, Brundage M, Cowan R, et al. Combined androgen deprivation therapy and radiation therapy for locally advanced prostate cancer: a randomised, phase 3 trial. Lancet. 2011;378:2104-11.

[8] Widmark A, Klepp O, Solberg A, Damber JE, Angelsen A, Fransson P, et al. Endocrine treatment, with or without radiotherapy, in locally advanced prostate cancer (SPCG-7/SFUO-3): an open randomised phase III trial. Lancet. 2009;373:301-8.

[9] Bolla M, Van Tienhoven G, Warde P, Dubois JB, Mirimanoff RO, Storme G, et al. External irradiation with or without long-term androgen suppression for prostate cancer with high metastatic risk: 10-year results of an EORTC randomised study. Lancet Oncol. 2010;11:1066-73.

[10] Fizazi K, Shore N, Tammela TL, Ulys A, Vjaters E, Polyakov S, et al. Darolutamide in Nonmetastatic, Castration-Resistant Prostate Cancer. N Engl J Med. 2019;380:1235-46.

[11] Norgaard M, Jensen AO, Jacobsen JB, Cetin K, Fryzek JP, Sorensen HT. Skeletal related events, bone metastasis and survival of prostate cancer: a population based cohort study in Denmark (1999 to 2007). J Urol. 2010;184:162-7.

[12] Coleman RE. Clinical features of metastatic bone disease and risk of skeletal morbidity. Clin Cancer Res. 2006;12:6243-9.

[13] Scher HI, Halabi S, Tannock I, Morris M, Sternberg CN, Carducci MA, et al. Design and end points of clinical trials for patients with progressive prostate cancer and castrate levels of testosterone: recommendations of the Prostate Cancer Clinical Trials Working Group. J Clin Oncol. 2008;26:1148-59.

[14] Boehmer D, Maingon P, Poortmans P, Baron MH, Miralbell R, Remouchamps V, Eortc radiation oncology group. Guidelines for primary radiotherapy of patients with prostate cancer. Radiother Oncol. 2006;79:259-69. 
[15] Mottet N, Bellmunt J, Bolla M, Briers E, Cumberbatch MG, De Santis M, et al. EAU-ESTRO-SIOG Guidelines on Prostate Cancer. Part 1: Screening, Diagnosis, and Local Treatment with Curative Intent. Eur Urol. 2017;71:618-29.

[16] Amling CL, Bergstralh EJ, Blute ML, Slezak JM, Zincke H. Defining prostate specific antigen progression after radical prostatectomy: what is the most appropriate cut point? J Urol. 2001;165:114651.

[17] Freedland SJ, Sutter ME, Dorey F, Aronson WJ. Defining the ideal cutpoint for determining PSA recurrence after radical prostatectomy. Prostate-specific antigen. Urology. 2003;61:365-9.

[18] Siddiqui SA, Boorjian SA, Inman B, Bagniewski S, Bergstralh EJ, Blute ML. Timing of androgen deprivation therapy and its impact on survival after radical prostatectomy: a matched cohort study. $\mathrm{J}$ Urol. 2008;179:1830-7.

[19] Duchesne GM, Woo HH, Bassett JK, Bowe SJ, D'Este C, Frydenberg M, et al. Timing of androgendeprivation therapy in patients with prostate cancer with a rising PSA (TROG 03.06 and VCOG PR 01-03 [TOAD]): a randomised, multicentre, non-blinded, phase 3 trial. Lancet Oncol. 2016;17:727-37.

[20] Moul JW, Wu H, Sun L, McLeod DG, Amling C, Donahue T, et al. Early versus delayed hormonal therapy for prostate specific antigen only recurrence of prostate cancer after radical prostatectomy. $J$ Urol. 2004;171:1141-7.

[21] van den Bergh RC, van Casteren NJ, van den Broeck T, Fordyce ER, Gietzmann WK, Stewart F, et al. Role of Hormonal Treatment in Prostate Cancer Patients with Nonmetastatic Disease Recurrence After Local Curative Treatment: A Systematic Review. Eur Urol. 2016;69:802-20.

[22] Algarra R, Hevia M, Tienza A, Merino I, Velis JM, Zudaire J, et al. Survival analysis of patients with biochemical relapse after radical prostatectomy treated with androgen deprivation: Castration-resistance influential factors. Can Urol Assoc J. 2014;8:333-41.

[23] Choueiri TK, Chen MH, D'Amico AV, Sun L, Nguyen PL, Hayes JH, et al. Impact of postoperative prostate-specific antigen disease recurrence and the use of salvage therapy on the risk of death. Cancer. 2010;116:1887-92.

[24] Keizman D, Huang P, Antonarakis ES, Sinibaldi V, Carducci MA, Denmeade S, et al. The change of PSA doubling time and its association with disease progression in patients with biochemically relapsed prostate cancer treated with intermittent androgen deprivation. Prostate. 2011;71:1608-15.

[25] Kim-Sing C, Pickles T, Prostate Cohort Outcomes, Initiative. Intervention after PSA failure: examination of intervention time and subsequent outcomes from a prospective patient database. Int $\mathrm{J}$ Radiat Oncol Biol Phys. 2004;60:463-9. 
[26] Moreira DM, Cooperberg MR, Howard LE, Aronson WJ, Kane CJ, Terris MK, et al. Predicting bone scan positivity after biochemical recurrence following radical prostatectomy in both hormone-naive men and patients receiving androgen-deprivation therapy: results from the SEARCH database. Prostate Cancer Prostatic Dis. 2014;17:91-6.

[27] Spratt DE, Zumsteg ZS, Pei X, Romesser PB, Yamada J, Kollmeier MA, et al. Predictors of castrationresistant prostate cancer after dose-escalated external beam radiotherapy. Prostate. 2015;75:175-82.

[28] Shipley WU, Desilvio M, Pilepich MV, Roach M, Wolkov HB, Sause WT, et al. Early initiation of salvage hormone therapy influences survival in patients who failed initial radiation for locally advanced prostate cancer: A secondary analysis of RTOG protocol 86-10. Int J Radiat Oncol Biol Phys. 2006;64:1162-7.

[29] Rodrigues NA, Chen MH, Catalona WJ, Roehl KA, Richie JP, D'Amico AV. Predictors of mortality after androgen-deprivation therapy in patients with rapidly rising prostate-specific antigen levels after local therapy for prostate cancer. Cancer. 2006;107:514-20.

[30] Hull GW, Rabbani F, Abbas F, Wheeler TM, Kattan MW, Scardino PT. Cancer control with radical prostatectomy alone in 1,000 consecutive patients. J Urol. 2002;167:528-34.

[31] Roehl KA, Han M, Ramos CG, Antenor JA, Catalona WJ. Cancer progression and survival rates following anatomical radical retropubic prostatectomy in 3,478 consecutive patients: long-term results. $J$ Urol. 2004;172:910-4.

\section{Tables}

Table 1. Clinicopathological characteristics of patients with localized prostate cancer who underwent radical prostatectomy and later developed metastasis. 


\begin{tabular}{|c|c|c|c|c|}
\hline & \multirow[t]{2}{*}{ Overall } & \multicolumn{2}{|c|}{ PSA at ADT } & \multirow[t]{2}{*}{$p$} \\
\hline & & $<2 \mathrm{ng} / \mathrm{mL}$ & $\geq 2 \mathrm{ng} / \mathrm{mL}$ & \\
\hline $\mathrm{N}$ & 69 & $31(45.0 \%)$ & $38(55.0 \%)$ & $N S$ \\
\hline Age (years) & $69.0(67.0-72.5)$ & $68.5(71.5-75.8)$ & $71.0(69.0-70.5)$ & 0.861 \\
\hline BMI $(\mathrm{kg} / \mathrm{m} 2)$ & $22.9(21.9-26.8)$ & $21.9(22.6-26.1)$ & $23.1(21.7-27.3)$ & 0.765 \\
\hline ECOG performance status & & & & 0.644 \\
\hline$\leq 1$ & $60(86.9 \%)$ & $27(87.1 \%)$ & $33(86.8 \%)$ & \\
\hline$\geq 2$ & $9(13.1 \%)$ & $4(12.9 \%)$ & $5(13.2 \%)$ & \\
\hline Preoperative PSA (ng/mL) & $17.4(7.4-50.0)$ & $20.0(9.3-43.0)$ & $14.8(8.6-75.5)$ & 0.256 \\
\hline PSA velocity & & & & 0.191 \\
\hline$\geq 2 \mathrm{ng} / \mathrm{mL} /$ year & $38(55.1 \%)$ & $16(51.6 \%)$ & $22(57.9 \%)$ & \\
\hline$<2 \mathrm{ng} / \mathrm{mL} /$ year & $31(44.9 \%)$ & $15(48.4 \%)$ & $16(42.1 \%)$ & \\
\hline PSA doubling time & & & & 0.246 \\
\hline$\geq 12$ months & $33(47.8 \%)$ & $14(45.2 \%)$ & $19(50.0 \%)$ & \\
\hline$<12$ months & $36(52.2 \%)$ & $17(54.8 \%)$ & $19(50.0 \%)$ & \\
\hline NCCN risk category & & & & 0.197 \\
\hline Low & $7(10.1 \%)$ & $5(16.2 \%)$ & $2(5.3 \%)$ & \\
\hline Intermediate & $18(26.1 \%)$ & $9(29.0 \%)$ & $9(23.7 \%)$ & \\
\hline High & $44(63.8 \%)$ & $17(54.8 \%)$ & $27(71.0 \%)$ & \\
\hline Pathologic Gleason score & & & & 0.582 \\
\hline$\leq 6$ & $13(18.8 \%)$ & $7(22.6 \%)$ & $6(15.8 \%)$ & \\
\hline 7 & $15(21.7 \%)$ & $8(25.8 \%)$ & $7(18.4 \%)$ & \\
\hline$\geq 8$ & $41(59.5 \%)$ & $16(51.6 \%)$ & $25(65.8 \%)$ & \\
\hline Pathological T stage & & & & 0.541 \\
\hline $\mathrm{T} 2$ & $12(17.4 \%)$ & $7(22.6 \%)$ & $5(13.1 \%)$ & \\
\hline T3 & $47(68.1 \%)$ & $20(64.5 \%)$ & $27(71.1 \%)$ & \\
\hline $\mathrm{T} 4$ & $10(14.5 \%)$ & $4(12.9 \%)$ & $6(15.8 \%)$ & \\
\hline Type of radiation therapy & & & & 0.158 \\
\hline Adjuvant & $11(15.9 \%)$ & $5(16.1 \%)$ & $6(15.8 \%)$ & \\
\hline Salvage & $58(84.1 \%)$ & $26(83.9 \%)$ & $32(84.2 \%)$ & \\
\hline
\end{tabular}

Data are median (interquartile range) and number (\%).

$\mathrm{ADT}=$ androgen-deprivation therapy; $\mathrm{BMI}=$ body mass index; CCI $=$ Charlson Comorbidity Index; $\mathrm{NCCN}=$ National Comprehensive Cancer Network; PSA = prostate-specific antigen

Table 2. Cox-regression models for the association of risk factors with progression to cancerspecific mortality. 


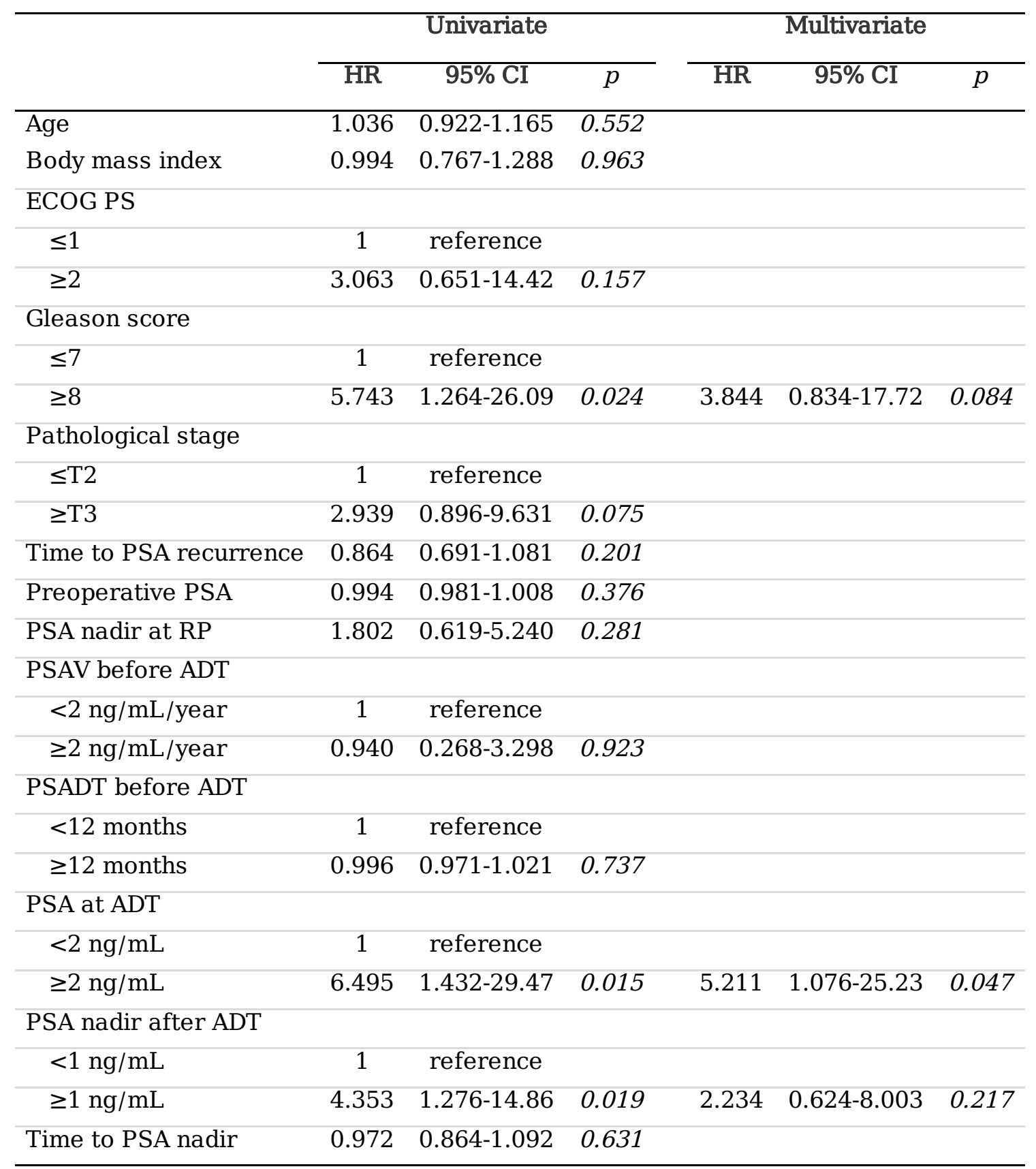

$\mathrm{ADT}=$ androgen-deprivation therapy $; \mathrm{CI}=$ confidence interval; ECOG PS $=$ Eastern Cooperative Oncology Group performance status; $\mathrm{HR}=$ hazards ratio; PSA = prostate-specific antigen; PSADT = prostate-specific antigen doubling time; PSAV = prostate-specific antigen velocity

Table 3. Cox-regression models for the association of risk factors with progression to castration-resistance. 


\begin{tabular}{|c|c|c|c|c|c|c|}
\hline & \multicolumn{3}{|c|}{ Univariate } & \multicolumn{3}{|c|}{ Multivariate } \\
\hline & $\overline{\mathrm{HR}}$ & $95 \% \mathrm{CI}$ & $p$ & HR & $95 \% \mathrm{CI}$ & $p$ \\
\hline Age & 0.951 & $0.884-1.023$ & 0.173 & & & \\
\hline Body mass index & 1.085 & $0.928-1.269$ & 0.306 & & & \\
\hline \multicolumn{7}{|l|}{ ECOG PS } \\
\hline$\leq 1$ & 1 & reference & & & & \\
\hline$\geq 2$ & 0.504 & $0.139-1.829$ & 0.298 & & & \\
\hline \multicolumn{7}{|l|}{ Gleason score } \\
\hline$\leq 7$ & 1 & reference & & & & \\
\hline$\geq 8$ & 2.202 & $0.894-5.424$ & 0.086 & & & \\
\hline \multicolumn{7}{|l|}{ Pathological stage } \\
\hline$\leq \mathrm{T} 2$ & 1 & reference & & & & \\
\hline$\geq \mathrm{T} 3$ & 0.779 & $0.168-3.607$ & 0.749 & & & \\
\hline Time to PSA recurrence & 0.938 & $0.870-1.011$ & 0.092 & & & \\
\hline Preoperative PSA & 1.004 & $0.994-1.015$ & 0.408 & & & \\
\hline PSA nadir at RP & 2.113 & $0.847-5.270$ & 0.109 & & & \\
\hline \multicolumn{7}{|l|}{ PSAV before ADT } \\
\hline$<2 \mathrm{ng} / \mathrm{mL} /$ year & 1 & reference & & & & \\
\hline$\geq 2 \mathrm{ng} / \mathrm{mL} /$ year & 1.487 & $0.604-3.657$ & 0.388 & & & \\
\hline \multicolumn{7}{|l|}{ PSADT before ADT } \\
\hline$<12$ months & 1 & reference & & & & \\
\hline$\geq 12$ months & 0.983 & $0.961-1.005$ & 0.134 & & & \\
\hline \multicolumn{7}{|l|}{ PSA at ADT } \\
\hline$<2 \mathrm{ng} / \mathrm{mL}$ & 1 & reference & & & & \\
\hline$\geq 2 \mathrm{ng} / \mathrm{mL}$ & 3.184 & $1.104-9.179$ & 0.032 & 3.934 & $0.994-15.57$ & 0.051 \\
\hline \multicolumn{7}{|l|}{ PSA nadir after ADT } \\
\hline$<1 \mathrm{ng} / \mathrm{mL}$ & 1 & reference & & & & \\
\hline$\geq 1 \mathrm{ng} / \mathrm{mL}$ & 3.792 & $1.286-11.19$ & 0.016 & 1.301 & $0.669-4.382$ & 0.511 \\
\hline Time to PSA nadir & 0.955 & $0.872-1.046$ & 0.324 & & & \\
\hline
\end{tabular}

$\mathrm{ADT}=$ androgen-deprivation therapy $\mathrm{CI}=$ confidence interval; ECOG PS = Eastern Cooperative Oncology Group performance status; HR = hazards ratio; PSA = prostate-specific antigen; PSADT = prostate-specific antigen doubling time; PSAV = prostate-specific antigen velocity

Table 4. Oncological outcomes of patients with localized prostate cancer who underwent radical prostatectomy and later developed metastasis. 


\begin{tabular}{lccc}
\hline & \multicolumn{2}{c}{ PSA at ADT } & $p$ \\
\cline { 2 - 3 } & $<2 \mathrm{ng} / \mathrm{mL}$ & $\geq 2 \mathrm{ng} / \mathrm{mL}$ & \\
\hline Time to metastasis (months) & $33.5(14.0-42.3)$ & $31.2(12.3-41.1)$ & 0.971 \\
Metastatic site & & & \\
\hline \multicolumn{1}{c}{ Bone } & $27(87.1 \%)$ & $33(86.8 \%)$ & 0.468 \\
\hline Lymph nodes & $3(10.0 \%)$ & $5(13.2 \%)$ & 0.621 \\
\hline Viscera & $2(6.5 \%)$ & $3(7.9 \%)$ & 0.133 \\
CRPC (\%) & $17(53.1 \%)$ & $30(51.7 \%)$ & 0.548 \\
Time to CRPC (months) & $55.5(35.2-97.8)$ & $42.5(32.3-73.8)$ & 0.051 \\
\hline CRPC-free progression, 5y (\%) & $70.8 \%$ & $64.7 \%$ & 0.311 \\
\hline Death, N (\%) & $7(21.9 \%)$ & $22(37.9 \%)$ & 0.001 \\
Time to CSM (months) & $72.0(45.0-108.9)$ & $65.0(52.5-90.0)$ & 0.045 \\
\hline CSS, 5y (\%) & $86.1 \%$ & $68.7 \%$ & 0.002 \\
\hline Follow-up (months) & $83.5(70.5-118.5)$ & $78(52.4-94.0)$ & 0.074 \\
\hline
\end{tabular}

Data are median (interquartile range) and number (\%).

$\mathrm{ADT}=$ androgen-deprivation therapy; $\mathrm{CRPC}=$ castration-resistant prostate cancer; $\mathrm{CSS}=$ cancer-specific survival; PSA = prostate-specific antigen

\section{Figures}

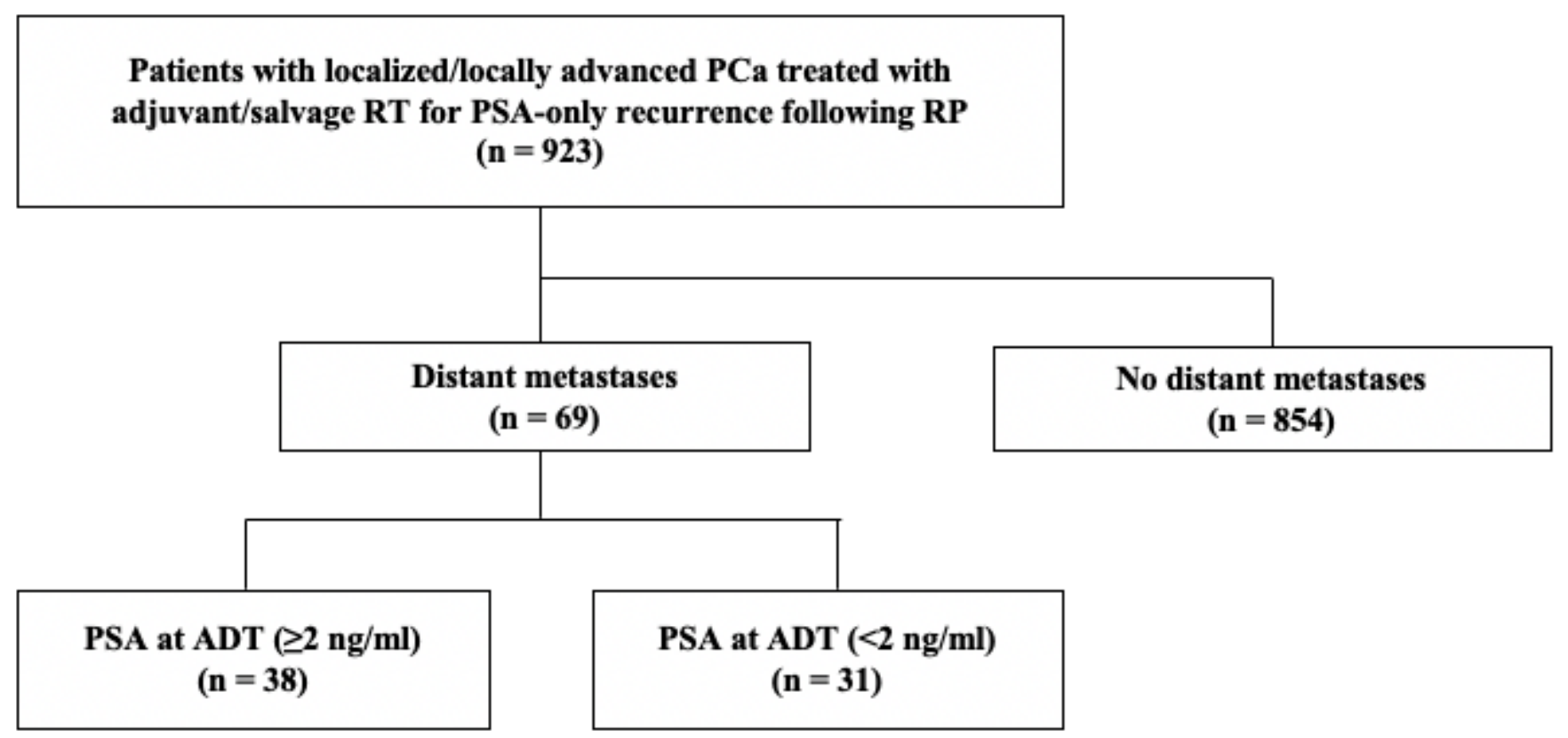

Figure 1

Flowchart of patient selection. 


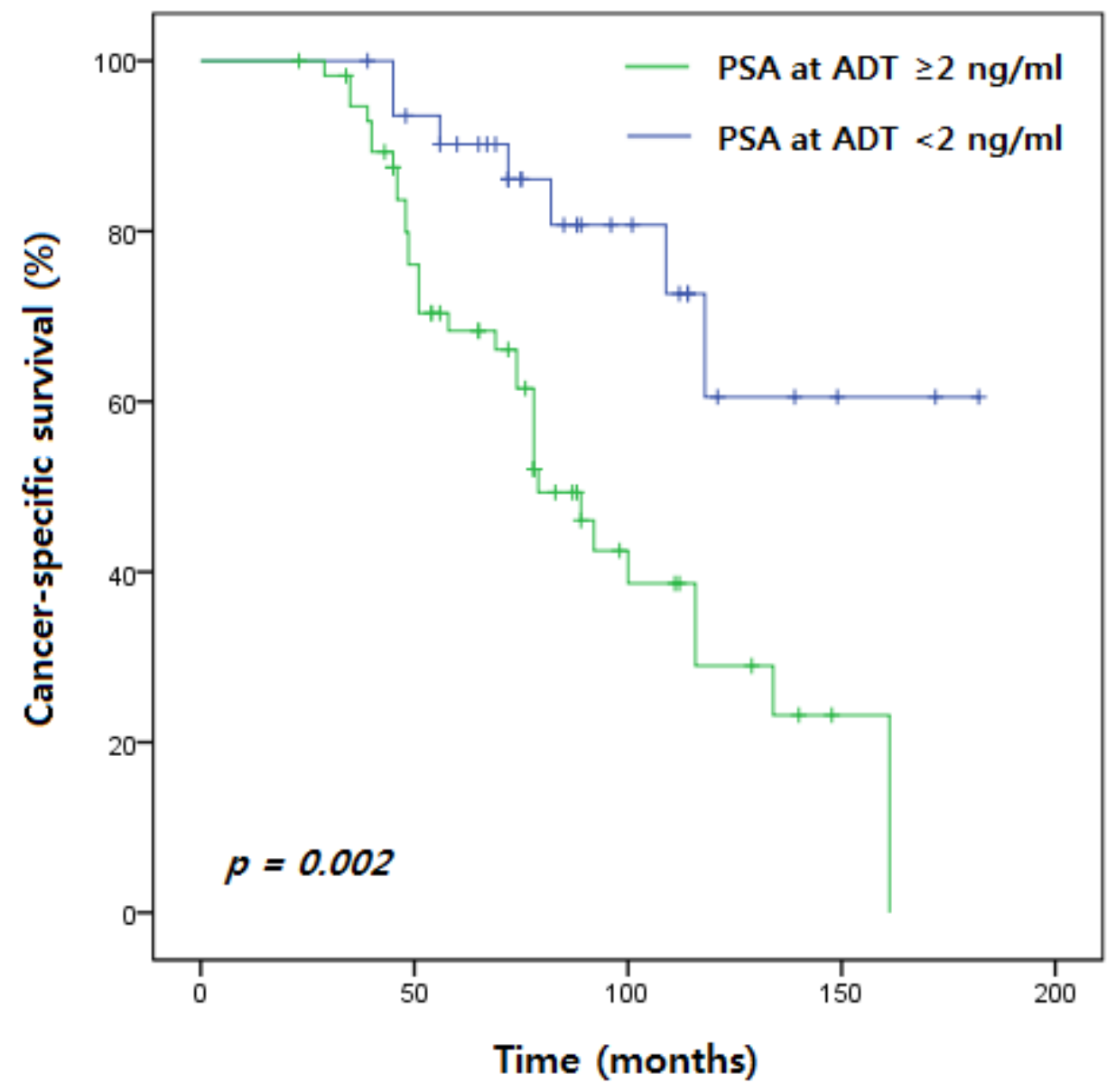

Figure 2

Kaplan-Meier curves showing cancer-specific survival, stratified by the PSA level at ADT of $2 \mathrm{ng} / \mathrm{mL}$. 


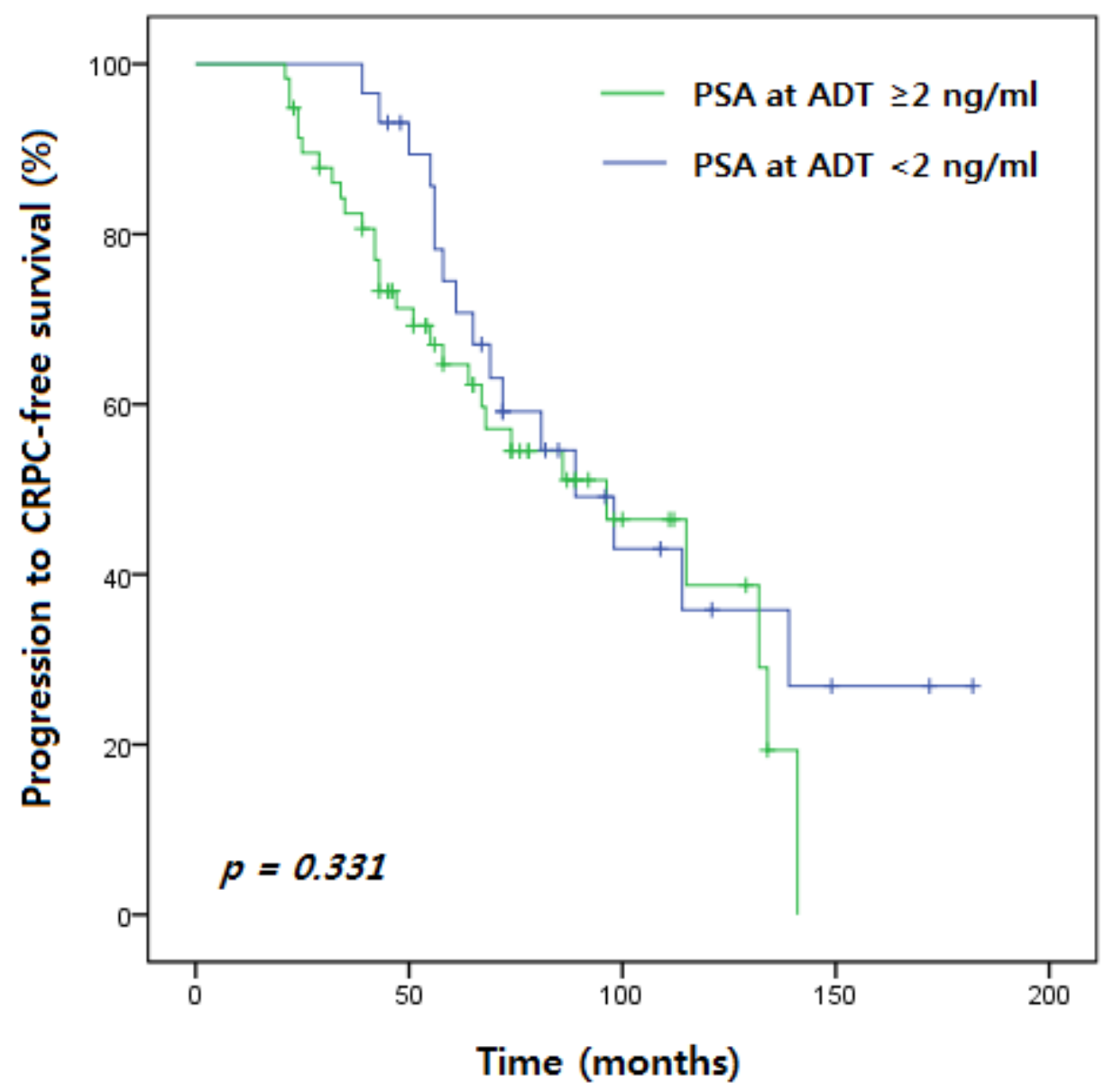

Figure 3

Kaplan-Meier curves showing with progression to castration-resistance prostate cancer-free survival, stratified by the PSA level at ADT of $2 \mathrm{ng} / \mathrm{mL}$. 